\title{
An Improved TIP Modeling Method
}

\author{
Lianjin Deng ${ }^{1}$, \\ ${ }^{1}$ Tianjin Bohai Vocational Technology College . \\ TianJin, China
}

\begin{abstract}
This paper presents the TIP superposition progression model, improves the traditional TIP modeling technology based on single image as a whole scene data sources, modeling of long scenes, the other end model will appear ambiguity phenomenon is very serious problem. This experiment adopts multiple photos, make up a single image information can be fully restored the whole scene shortcomings, the abundant information from multiple pictures, vividly reproduce the whole street. Accordingly, the use of multi image registration, fusion, extraction of useful information, and then texture mapping, the final completion of the depth of the big scene modeling.
\end{abstract}

Keywords: TIP model, IBR

\section{INTRODUCTION}

As a branch of virtual reality technology, image based rendering (Image Based Rendering, IBR) technology, which is a group of photos into the real world, after processing, the image to get a new viewpoint. It overcomes the traditional geometric modeling method based on the shortcomings, has higher rendering efficiency, and has real effects picture level. The middle painting (Tour Into the Picture, TIP) as a specific IBR realization method, is put forward by Horry in 1997, it as long as the input of a single image, the application of the principle of projection geometry, only can process simple to generate realistic 3D scenes, and can roam in the.

\section{IMAGE MATCHING}

\section{A. Feature points extraction}

Received date: -- Deng Lianjin, 1981, female, master, lecturer, artificial intelligence and graphics and image; You Dexiang, male, 1982, master. Engineers, graphic image

Image corner refers to the image edge line curvature of the local maximum curvature, also can be greater than a certain threshold point. Because the image corner points is far less than the total number of pixels, so as the feature point. Feature points detection method of very many of the existing, here the use of classical Harris operator for image feature point extraction, TIP model of seamless connection and lay the foundation for. Its principle is as follows:

Assuming the image brightness value of I (x, y), w (x, y window template) after small offset $(\mathrm{U}, \mathrm{V})$, pixel $(\mathrm{x}, \mathrm{y})$ of the brightness variation is denoted as $\mathrm{E}(\mathrm{U}, \mathrm{V})$, then Now you can type your paper, or copy the old version of your paper onto this new formated file.

\author{
Dexiang $\mathrm{You}^{2}$ \\ ${ }^{2}$ The Third Railway Survey and Design Institute . \\ TianJin, China
}

$$
\begin{gathered}
E(u, v)=\left[\begin{array}{ll}
u & v
\end{array}\right] M\left[\begin{array}{l}
u \\
v
\end{array}\right] \\
M=\left(\begin{array}{ll}
A & C \\
C & B
\end{array}\right)=w(x, y) \otimes\left(\begin{array}{cc}
I_{x}^{2} & I_{x} I_{y} \\
I_{x} I_{y} & I_{y}^{2}
\end{array}\right)
\end{gathered}
$$

$I_{x} I_{y}$ respectively derivative pixel along the $\mathrm{X}, \mathrm{y}, \mathrm{w}$ use Gaussian function in order to better deal with noise.

If the results of the two eigenvalues of matrix $M$ has a local maximum, the corner. In order to avoid the eigenvalue calculation, selection of evaluation function

$$
R=M_{d}-k M_{t}^{2}
$$

Type $M_{d}$ said the value of the determinant of the matrix $\mathrm{M}, M_{t}$ matrix representation of $\mathrm{M}$ trace, The feature points corresponding points for $\mathrm{R}$ exceeds $\mathrm{a}$ threshold, the threshold number of feature points decided by. Decision reliable probabilistic robustness algorithm the number of feature points from the described below. Below is the Harris corner detection on campus in a corner of the results.

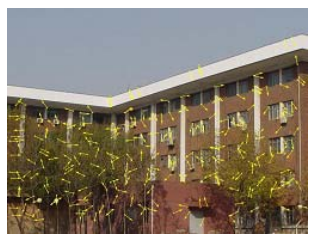

Figure 1 Harris corner detection results

\section{B. Feature matching}

Corner the algorithm to obtain the distribution in the whole image, and because the image the presence of noise, so that the corner detection errors exist. Corner here using entropy positioning accuracy of screening has been obtained, leave can be adjacent image overlap region registration those corner points, and then by the bidirectional optimal matching criterion to find the best matching point set. 


\section{1) Entropy transform}

Entropy is used to measure the complexity of a system and uncertainty. The size of image entropy can be defined as:

$$
H_{f}=-\sum_{i=1}^{m} \sum_{j=1}^{n} P_{i, j} \log P_{i, j}
$$

Type $p_{i, j}$ is the image intensity distribution, can be expressed as:

$$
p_{i, j}=f(i, j) / \sum_{i=1}^{m} \sum_{j=1}^{n} f_{i, j}
$$

By formula (3) shows, entropy reflects the image contains the information of size, if the image gray scale change drastically, with larger entropy, and the gray change is less uniform, entropy. Image local entropy is a plurality of pixel local window in the results, can not be affected by the single point noise, which can play a role of filtering.

The observation that pixel $f(i, j)$ has the same contribution to local entropy. In order to make the contribution of pixel distance of the center of local entropy $f(u, v)$ closer to the entropy is bigger, the introduction of $d(i, j)$ as a measure of Euclidean distance:

$$
d(i, j)=\left[(i-u)^{2}+(j-v)^{2}\right]^{1 / 2}
$$

$d(i, j)$ says $f(i, j)$ the distance of the center distance of local entropy $f(u, v)$. The type of rewriting (3)

$$
H_{f}=-\sum_{i=1}^{m} \sum_{j=1}^{n} \frac{1}{d(i, j)} \bullet p_{i, j}\left(1-p_{i, j}\right)
$$

Type (5) solving process become entropy image transform.

2) Find the match set of points.

The basic steps of the algorithm:

1) Image feature point extraction using Harris operator, has the focus of adjacent image set respectively:

a) On in every corner as the center, transform image entropy calculation module, module size can choose $3 * 3$, $5 * 5$, or $7 * 7$;

b) On in every corner of, in search with corner minimum entropy difference, get the matching corner sets ;

c) On in every corner of, in search with corner minimum entropy difference, get the matching corner sets ;

d) Calculate best matched point set

The algorithm first performs image entropy transform, and then uses the bidirectional matching method to obtain the best matched point set, so as to realize the image registration based on feature points.
If there are no further authors place the cursor one space behind the word ABSTRACT: and type your abstract of not more than 150 words. The top of the first line of the abstract will be $73 \mathrm{~mm}$ (2.87") from the top of the type area. The complete abstract will fall in the abstract frame, the settings of which should also not be changed (Width: Exactly 187 mm (7.36"); Height: Automatic; Vertical 73 mm (2.87") from margin; Lock anchor).

\section{ROBUST ESTIMATION OF THE TRANSFORMATION MATRIX}

On two sites along the same direction, the image translation view shooting, the corresponding matching points between meet the following transformation: Layout of text

$$
\left[\begin{array}{c}
x^{\prime} \\
y^{\prime} \\
s
\end{array}\right]=\left[\begin{array}{lll}
H_{11} & H_{12} & H_{13} \\
H_{21} & H_{22} & H_{23} \\
H_{31} & H_{32} & H_{33}
\end{array}\right]\left[\begin{array}{l}
x \\
y \\
1
\end{array}\right]=H\left[\begin{array}{l}
x \\
y \\
1
\end{array}\right]
$$

Type (6) integration transform has 8 independent parameters, only need at least 4 feature point pairs, and any 3 noncollinear points, can only determine the transformation matrix $\mathrm{H}$ 。

Adjacent image section calculated matching point set based method, using the least mean two times estimated can obtain the transformation matrix $\mathrm{H}$ 。 The specific algorithm is as follows:

1) For each random minimal subset (4 characteristics of type 6) according to the calculated $\mathrm{H}$, then of all feature points is calculated and the median error:

For the corresponding feature points, $\mathrm{d}$ is the Euclidean distance.

The repeated execution of $\mathrm{L}$, corresponding to the minimum value of written under the $\mathrm{H}$; In order to reduce the effects of noise, robust estimation of variance calculation

Calculating the weights of, including according to step 3 of the weight of, by using the weighted least squares method to calculate new values: two pieces of type (6) adjacent image after transformation, then the projection plane, get the texture image of each wall in the TIP model, now there are still some ghost image overlap, must through the fusion can achieve seamless connection the effect of.

\section{THE SUPERPOSITION OF PROGRESSIVE TIP MODEL}

Here presents a new model of TIP connection method. In a long channel along the end of shooting to the other end, see Figure 2, after a picture of the contents of the B must be a subset of the previous picture of the contents of the $\mathrm{A}$, if the content of the image after a deformation, which mapped to a rectangular region $\mathrm{B}$ 'previous image, then can be seen from position $\mathrm{A}$ to position $\mathrm{B}$ time, the picture area was gradually enlarged to become B B'. It can be concluded that, if the rectangular $\mathrm{B}$ 'as photos, the photos back TIP model established by A using B to fill in, instead of the usual B', 
can effectively solve the problem of long channel TIP model fuzzy back, because from the long channel one end to the other end of the interval is not the photos taken on, down about four wall length is not too long, the fuzzy phenomenon is not obvious, so that it can be connected with the implementation of TIP, as long as the modeling of the photo $\mathrm{Ai}$ using $\mathrm{Ai}+1$ instead of $\mathrm{Ai}$ in the $\mathrm{Ai}+1$ region can'. Another advantage of doing this is not within the user specified rectangle.

It just takes two images as an example, when in the long channel shooting multi image modeling, can start from an image of the recent continuous extraction, after an image as the previous image formed in rectangular, TIP model and so on, finally establish the whole scene, as shown in figure

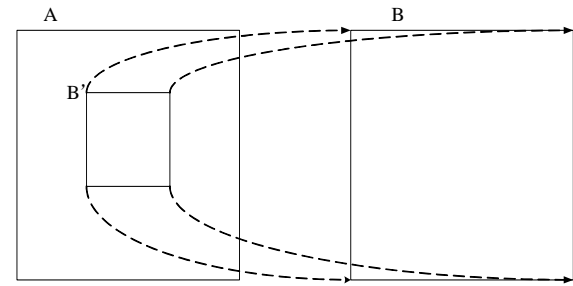

Figure 2 Adjacent mapping between two images

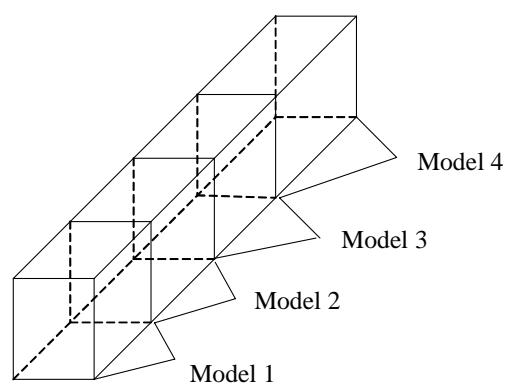

Figure 3 Multiple images of the TIP model to connect

\section{A. The algorithm steps are as follows:}

1) Acquiring an image sequence $A[1 \sim n]$, a fusion result mapFront [1 n-1] and Back, the initial Back=A[1], variable $i=1$;

2) On Back and $A[i+1]$ image feature matching, getRectangular $A[i+1]$ ' $A[i+1]$ in the Back corresponding to the Back, and the $A[i+1]$ amplification to make' $A[i+1]$ as large, then the wavelet image fusion, the fusion image is obtained by the Chinese and foreign rectangular and Front is a new [i], a rectangle in Back;

3) $i++$, if $i>=n$ then go to step (4), or go to (2);

4) On Front $[1 \sim n]$ multi resolution extraction, obtains $a$ series The same size, image zooming along the line of sight, and then respectively by TIP modeling, finally carries on the connection you can get the TIP model of long distan

\section{SUMMARY AND PROSPECT}

This article carries on the further study of TIP modeling theory, and put forward the method of connecting multiple
TIP model, solve the practical problem often encountered in the process of large-scale scene modeling, as shown in Figure 4, is a single TIP model deep when roaming effect increases with depth, roaming, appear obvious fuzzy phenomenon, and figure 5 using the multi TIP model to construct the virtual scenes, still keep the scene definition better in depth gradually increase.

The information of multiple images fusion method is adopted in this paper, which makes the TIP modeling upgrade from a single data source to multiple data sources, so as to realize the long channel TIP modeling, which laid the foundation for the application of IBR in the field of large scale scene roaming, ultimately to achieve large-scale scene image based on modeling.

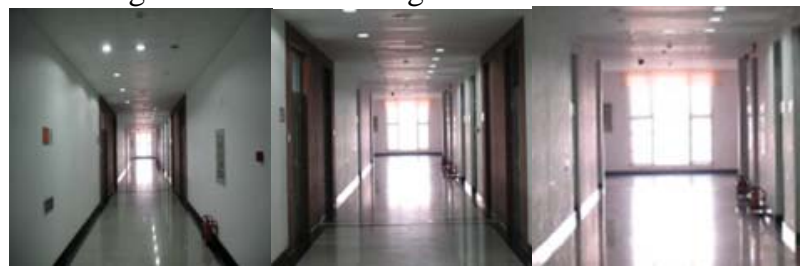

Figure 4 TIP model of single-depth roaming screenshot

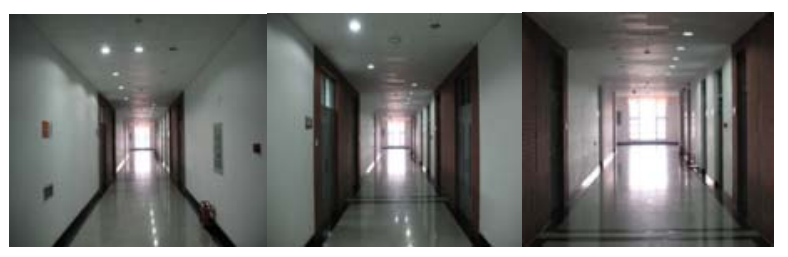

Figure 5 TIP model more depth smooth connected roaming screenshot

\section{REFERENCES}

[1] Shum H Y, Kang S B. A review of image-based rendering techniques [A]. In: Proceedings of IEEE/SPIE Visual Communications and Image Processing, Perth, Australia, 2000. 2-3

[2] Youichi Horry, Ken-ichi Anjyo, Kiyoshi Arai, Tour Into the Picture: Using a Spidery Mesh Interface to

[3] Make Animation from a Single Image [A]. In: Proceedings of the SIGGRAPH'97, Los Angeles, LA,USA,1997. 225-232

[4] Eun-Young (Elaine) Kang, Ilmi Yoon, Smooth Scene Transition for Virtual Tour on the World Wide Web[C]. ICCIMA 2005,August 2005.Los Alamitos,California,USA:219-225.

[5] Yuebing Wu, Research of Virtual Environment Rendering Based on Image and Geometry[A]. In: Proceedings of Third International Conference on Image and Graphics (ICIG'04), 2004.511-514

[6] Qiushuang Zhang, Kan Zhao, Zhiqing Cao,etc.An Efficient Imagebased Virtual Tour System [Z]

[7] Paul Ernest Debevec, Modeling and Rendering Architecture from Photographs : A hybid geometry and image based approach[A]. In: Proceedings of the 23rd annual conference on Computer graphics and interactive techniques , 11 - 20PaulDebevec, YizhouYu, GeorgeBorshukov, Efficient View-Dependent Image-Based Rendering with Projective Texture-Mapping [A]. In: Proceedings of the 9th Eurographics Workshop on Rendering, Vienna, 1998. 105116

[8] Sanghyuk Yoon, Hai-jung Chen, Tom Hsu ,etc, Web-based Virtual Tour Using The Tour Into The Picture

[9] (TIP) Technique[A]. In: Proceedings of th International Conference on Distributed Multimedia Systems,2003: 81-88. 